\title{
Dishonest Newton Method Applied in Continuation Power Flow through a Geometric Parameterization Technique
}

\author{
A. Bonini Neto, E. M. Magalhães and D. A. Alves
}

\begin{abstract}
The electricity sector has gone through several transformations due primarily to increased electricity demand, especially in emerging countries. This encourages more investment in the generation and leads to a growing concern about the development and improvement of static voltage stability tools of power systems. This paper presents a geometric parameterization technique for continuation power flow based on the addition of a line which passes through of points in the plane formed by the variables of voltage magnitude or voltage angle and loading factor. This technique eliminates the Jacobian matrix singularity at the maximum loading point, which allows obtaining the solution trajectory ( $\mathrm{P}-\mathrm{V}$ curve) without any need to change the parameter, which is a very common procedure in the continuation power flow currently available. This paper also investigates the influence of updating the modified Jacobian matrix only when the system undergoing significant change (Dishonest Newton Method), rather than update it every iteration. The results show a reduction of the CPU time in tracing of $\mathrm{P}-\mathrm{V}$ curve.
\end{abstract}

Keywords - P-V curve, Continuation power flow, Jacobian matrix, Maximum loading point, Parameterization technique.

\section{NOMENCLATURA E SIMBOLOGIA}

PMC - Ponto de máximo carregamento

FCC - Fluxo de carga continuado

$\mathrm{P}-\mathrm{V}$ - Curva tensão versus carregamento

FC - Fluxo de carga

FCCP - fluxo de carga continuado proposto

$\mathrm{MC}$ - Margem de carregamento

PM - Ponto médio

$\boldsymbol{\theta}$ - Vetor dos ângulos de fase nodais

$\mathbf{V}$ - Vetor das magnitudes de tensões nodais

J - Matriz Jacobiana

$\mathbf{J}_{\mathbf{m}}$ - Matriz Jacobiana modificada

$\mathbf{G}$ - Vetor composto pelas equações de balanço de potência ativa e reativa das barras do sistema

$\Delta \mathbf{P}-$ Mismatches de potência ativa

$\Delta \mathbf{Q}$ - Mismatches de potência reativa

$\mathbf{P}_{\mathbf{g}}^{\mathbf{s p}}-$ Vetor de potência ativa gerada

$\mathbf{Q}_{\mathrm{g}}-$ Vetor de potência reativa gerada

$\mathbf{P}_{\mathbf{c}}{ }^{\text {sp }}$ - Vetor de potência ativa consumida

Os autores agradecem ao apoio financeiro do CNPq e Capes.

A. Bonini Neto, Departamento de Engenharia de Biossistemas, UNESP, Tupã, Brasil, bonini@tupa.unesp.br

E. M. Mabalhães, Departamento de Engenharia Elétrica, UNESP, Ilha Solteira, Brasil, elisabete.magalhães@yahoo.com.br

D. A. Alves, Departamento de Engenharia Elétrica, UNESP, Ilha Solteira, Brasil, dalves@dee.feis.unesp.br
$\mathbf{Q}_{\mathbf{c}}{ }^{\mathbf{s p}}-$ Vetor de potência reativa consumida

$\mathbf{P}^{\mathbf{s p}}$ - Vetor da diferença entre os vetores de potência ativa gerada e consumida

$\mathbf{Q}^{\mathbf{s p}}$ - Vetor da diferença entre os vetores de potência reativa gerada e consumida

PV - Barra de geração

PQ - Barra de carga

$\lambda$ - Fator de carregamento

$\alpha$ - Parâmetro de continuação que determina a nova equação quadrática

$\Omega$ - Conjunto de todas as barras da rede

$g_{k m}$ - Condutância série do ramo entre as barras k e m

\section{INTRODUÇÃO}

A O longo dos anos, o mercado de eletricidade em geral tem experimentado um aumento sensível da demanda de energia associado a uma série de reformas e reestruturações das empresas de energia elétrica, o que tem conduzido a um aumento na concorrência entre as empresas do setor [1]. A redução do tempo de CPU dos algoritmos utilizados para a obtenção das curvas $\mathrm{P}-\mathrm{V}$ nas fases de planejamento e operação de um Sistema Elétrico de Potência é de grande interesse por parte das empresas. Esse interesse é devido à necessidade de se definir margens de estabilidade estática de tensão e ações de controle indispensáveis não só para as condições do caso base (condições normais de operação), mas também para diferentes condições de contingência [22]. Assim, as principais características esperadas para o método de fluxo de potência a ser utilizado são a eficiência computacional e a robustez. Nesse sentido os métodos de fluxo de carga continuado (FCC) através do algoritmo de Newton-Raphson mostram-se como os mais robustos. O FCC realiza o traçado completo das curvas $\mathrm{P}-\mathrm{V}$ através da variação automática do valor de um determinado parâmetro do sistema. A sua robustez é devida à reformulação das equações do FC a qual a singularidade da matriz Jacobiana (J) é eliminada no PMC e, consequentemente, os problemas numéricos que ocorrem em torno deste. A remoção da singularidade é, em geral, efetuada por meio da adição de equações parametrizadas. Várias técnicas de parametrização e obtenção do PMC têm sido apresentadas na literatura [2-12]. Apesar disso, em virtude da necessidade de redução do tempo computacional, qualquer desenvolvimento adicional objetivando obter uma melhora nas características de convergência do processo de solução, bem como uma redução do tempo computacional necessário para o traçado da curva P- 
$\mathrm{V}$ tem representado um campo de estudo muito atrativo [12$22]$.

Nos algoritmos de FCC, a cada iteração, um conjunto de equações não lineares é linearizado em torno da solução atual, e a atualização do estado é obtida pela solução destas equações. Assim, nesse procedimento, a cada iteração os elementos pertencentes à matriz Jacobiana são atualizados. Contudo, após vários estudos realizados, concluiu-se que a matriz Jacobiana é importante para a convergência do processo, mas isso não influencia a solução final. Observe que embora as correções a cada iteração sejam aproximações, o valor da função é calculado de forma exata. Em geral, o processo iterativo parte de uma boa estimativa inicial, ou seja, o próximo ponto de operação é obtido ou a partir do ponto anterior, no caso do preditor trivial ou secante de ordem zero, ou a partir de uma estimativa obtida pelo passo preditor, nos casos dos preditores tangente e secante de primeira ordem [5], [19] e [23]. Dessa forma, a robustez do método é tal que é possível se considerarem aproximações na matriz $\mathbf{J}$ sem que se perca a convergência global, e isso, à custa apenas de um aumento relativamente pequeno do número de iterações. A convergência desses métodos também é afetada pelo ajuste das soluções em decorrência da violação dos limites de potência reativa nas barras de geração (barras PV) é outro ponto que ficou claro. Assim, no tocante a eficiência computacional, um procedimento comumente utilizado nos métodos de FC e denominado aqui como P2 é o de atualizar a matriz Jacobiana somente quando o sistema sofrer alguma mudança significativa (p.ex., quando da mudança no tipo da barra PV para PQ em virtude da violação de seus limites) e não a cada iteração. Esse procedimento conhecido como Dishonest Newton Method [24], muitas vezes possibilita um ganho considerável no tempo de processamento.

Assim, esse trabalho tem por objetivo apresentar os resultados da avaliação da técnica de parametrização local inicializada em [9], comparando seu desempenho considerando dois procedimentos, P1 e P2. Outra ideia proposta neste trabalho é apresentar o plano $\lambda$ - $\theta$ para o traçado da curva P-V. Para este trabalho, a junção do método inicializado em [9] com os procedimentos aqui utilizados mais o uso do plano $\lambda-\theta$, dar-se-á o nome de fluxo de carga continuado proposto (FCCP). No primeiro procedimento (P1) em que a atualização da $\mathbf{J}$ é realizada a cada iteração e no segundo $(\mathrm{P} 2)$, em que visa à redução do tempo computacional necessário para obtenção da curva $\mathrm{P}-\mathrm{V}$, atualiza-se a matriz somente quando o sistema sofrer alguma mudança significativa.

Os sistemas utilizados para o desempenho do método utilizando matrizes constantes para o traçado das curvas P-V são os sistemas IEEE 14, 118 e 300-barras, uma configuração de 638 barras do sistema Sul-Sudeste Brasileiro e sistema de 904 barras localizado no Sudoeste Americano.

\section{FLUXO DE CARGA CONTINUADO}

$\mathrm{O}$ traçado da curva $\mathrm{P}-\mathrm{V}$ é realizado por meio de sucessivas soluções do sistema de equações do FC, considerando um crescimento da carga numa direção predefinida. De forma a se automatizar o processo de levantamento da curva $\mathrm{P}-\mathrm{V}, \mathrm{O}$ conjunto de equações do FCC, na sua forma mais geral, tem a seguinte forma:

$$
\mathbf{G}(\boldsymbol{\theta}, \mathbf{V}, \lambda)=\mathbf{0}
$$

e que pode ser reescrita como:

$$
\begin{aligned}
& \Delta \mathbf{P}(\boldsymbol{\theta}, \mathbf{V}, \lambda)=\mathbf{P}^{\mathrm{sp}}(\lambda)-\mathbf{P}(\boldsymbol{\theta}, \mathbf{V})=\lambda\left(\mathbf{P}_{\mathbf{g}}^{\mathrm{sp}}-\mathbf{P}_{\mathbf{c}}^{\mathrm{sp}}\right)-\mathbf{P}(\boldsymbol{\theta}, \mathbf{V})=\mathbf{0} \\
& \Delta \mathbf{Q}(\boldsymbol{\theta}, \mathbf{V}, \lambda)=\mathbf{Q}^{\mathrm{sp}}(\lambda)-\mathbf{Q}(\boldsymbol{\theta}, \mathbf{V})=\mathrm{Q}_{\mathbf{g}}-\lambda\left(\mathbf{Q}_{\mathbf{c}}^{\mathbf{s p}}\right)-\mathbf{Q}(\boldsymbol{\theta}, \mathbf{V})=\mathbf{0}
\end{aligned}
$$

onde $\lambda$ é o fator de carregamento; $\mathbf{V}$ e $\boldsymbol{\theta}$ são os respectivos vetores de magnitude de tensão nodal e ângulo das fases, respectivamente; $\mathbf{P}^{\mathbf{s p}}(\lambda)$ é o vetor da diferença entre os vetores de potência ativa gerada $\left(\mathbf{P}_{\mathbf{g}}^{\mathbf{s p}}\right)$ e consumida $\left(\mathbf{P}_{\mathbf{c}}{ }^{\text {sp }}\right)$ especificada para as barras de carga $(P Q)$ e geração $(P V)$; e $\mathbf{Q}_{\mathbf{c}}{ }^{\mathbf{s p}}(\lambda)$ é o vetor de potência reativa consumida especificada para as barras $P Q$.

A equação (1) assume que o carregamento da rede é proporcional ao do caso base e considera o fator de potência constante. $\mathbf{P}^{\mathrm{sp}}(\lambda)$ e $\mathbf{Q}^{\mathrm{sp}}(\lambda)$ também podem ser definido como sendo igual a $\lambda\left(\mathbf{k}_{\mathrm{Pg}} \mathbf{P}_{\mathrm{g}}{ }^{\text {sp }}+\mathbf{k}_{\mathrm{Pc}} \mathbf{P}_{\mathbf{c}}{ }^{\text {sp }}\right)$ e $\lambda \mathbf{k}_{\mathrm{Qc}} \mathbf{Q}_{\mathbf{c}}{ }^{\text {esp }}$, respectivamente. Os vetores $\mathbf{k}_{\mathrm{Pg}}$, $\mathbf{k}_{\mathrm{Pc}}$ e $\mathbf{k}_{\mathrm{Qc}}$ são parâmetros fixos usado para caracterizar um específico cenário de carga.

$\mathrm{O}$ traçado da curva $\mathrm{P}-\mathrm{V}$ pode ser feito por sucessivas soluções de (1), utilizando um FC. O PMC é obtido através da adoção de $\lambda$ como um parâmetro. Seu valor é aumentado gradualmente a partir do caso base $(\lambda=1)$, até um valor para o qual nenhuma solução seja obtida, ou seja, para qual o processo iterativo do fluxo de potência não convirja ou divirja. Em geral, neste ponto, haverá um controle de passo consistindo de uma simples redução no incremento (tamanho do passo) de $\lambda$ e a nova solução é obtido a partir da última solução convergente. O PMC é considerado o último ponto convergido após sucessivas repetições deste procedimento. No entanto, a divergência do FC é uma consequência da singularidade da matriz Jacobiana (J) de (1) no PMC e, portanto, seu valor não pode ser determinado com precisão. A fim de superar as dificuldades numéricas resultantes da singularidade de $\mathbf{J}$ e permitir a determinação do $\mathrm{PMC}$, foram propostas diferentes parametrizações para o método da continuação [5-8], [23] e [25]. O método da continuação é constituído de quatro elementos básicos: o procedimento de parametrização, o passo preditor, o controle de passo e o passo corretor [2], [10] e [26-28]. A parametrização fornece um modo para identificar cada uma das soluções ao longo da trajetória de solução a ser obtida.

Para traçar a curva $\mathrm{P}-\mathrm{V}$ visando à obtenção do $\mathrm{PMC}$ e consequentemente, da margem de carregamento, é adicionado a (1) uma equação da reta, ou seja, uma função polinomial do primeiro grau que passa através de dois pontos no plano definido pelo fator de carregamento $(\lambda)$ e a magnitude da tensão $\left(\mathrm{V}_{\mathrm{k}}\right)$ ou ângulo da tensão $\left(\theta_{\mathrm{k}}\right)$ de uma barra $k$ : 


$$
R(\mathbf{y}, \lambda, \alpha, \beta)=\alpha\left(\lambda-\lambda^{0}\right)-\beta\left([\mathbf{y}]-\left[\mathbf{y}^{0}\right]\right)=0
$$

em que $\alpha$ e $\beta$ são coeficiente angulares que definem a reta a ser utilizada, e $[\mathbf{y}]$ é a medida escalar do vetor $\mathbf{y}=\left[y_{1}, \ldots, y_{n}\right]^{\mathrm{T}}$, a qual pode ser escolhida dentre várias formas [3] e [14]:

$[\mathbf{y}]=y_{k}$ onde $k$ é qualquer um dos índices $1 \leq k \leq n$;

$[\mathbf{y}]=\|\mathbf{y}\|_{\infty}=$ máx. $\left\{\left|y_{1}\right|,\left|y_{2}\right|, \ldots,\left|y_{\mathrm{n}}\right|\right\}$

$$
[\mathbf{y}]=\|\mathbf{y}\|_{3}=\left(y_{1}+y_{2}+\ldots+y_{\mathrm{n}}\right) / \mathrm{n}=\left(\Sigma y_{k}\right) / \mathrm{n}
$$

As duas primeiras formas encontram-se dentre as técnicas de parametrização local, enquanto que a última é um exemplo da técnica de parametrização global.

\subsection{Fluxo de carga continuado proposto (FCCP)}

O FCCP utiliza a equação de uma reta que passa através de um ponto no plano determinado pelas variáveis fator de carregamento $(\lambda)$ e magnitude da tensão nodal ou ângulo da tensão de uma barra $k$ qualquer. Assim, considera-se $[\mathbf{y}]=y_{k}$ onde $k$ é qualquer um dos índices $1 \leq k \leq n, n$ o número de barras. Conforme é apresentado na Fig. 1 , o valor de $\beta=1$ e o parâmetro $\alpha$ é o coeficiente angular da reta que passa por um ponto inicial escolhido, ponto "O" $\left(\lambda^{0}=0,[y]^{0}=V_{k}=0.7\right)$ ou $\left(\lambda^{0}=0,[\mathbf{y}]^{0}=\theta_{k}=\theta_{\mathrm{cb}}\right)$, situado no plano formado pelas variáveis $\lambda$ e [y] onde $\theta_{\mathrm{cb}}$ é o valor do ângulo no caso base. Com a adição de uma dessas equações, $\lambda$ pode ser tratado como uma variável dependente e $\alpha$ como variável independente, ou seja, $\alpha$ pode ser escolhida como parâmetro. Com isso, o número de incógnitas é igual ao de equações, isto é, uma condição necessária para que se tenha solução é atendida, desde que a matriz tenha posto completo, isto é, seja não singular.

Assim, com a solução do caso base obtida $\left(\boldsymbol{\theta}^{1}, \mathbf{V}^{1}\right.$ e $\left.\lambda^{1}\right)$ calcula-se o valor de $\alpha$ a partir do ponto inicial escolhido "O" e dos seus respectivos valores obtidos no caso base "P" $\left(\lambda^{1}\right.$, $\left.[\mathbf{y}]^{1}\right)$ :

$$
\alpha^{1}=\left([\mathbf{y}]^{1}-[\mathbf{y}]^{0}\right) /\left(\lambda^{1}-\lambda^{0}\right)
$$

Para $\alpha=\alpha^{1}+\Delta \alpha$, a solução do novo sistema de equações formado pelas equações (1) e (3) fornecerá o novo ponto de operação $\left(\theta^{2}, \mathbf{V}^{2}\right.$ e $\left.\lambda^{2}\right)$ correspondente à interseção da trajetória de soluções (curva $\lambda$-[y]) com a reta cujo novo valor de coeficiente angular $\left(\alpha^{1}+\Delta \alpha\right)$ foi especificado. Para $\alpha=\alpha^{1}$, a solução obtida deverá resultar em $\lambda=1$.

Expandindo o novo sistema de equações em série de Taylor, incluindo somente os termos de primeira ordem e considerando o valor prefixado no valor do parâmetro $\alpha$ calculado para o caso base, resulta em:

$$
-\left[\begin{array}{cc}
\mathbf{J} & \mathbf{G}_{\lambda} \\
\partial \mathrm{R} / \partial \mathrm{x} & \alpha
\end{array}\right]\left[\begin{array}{c}
\Delta \mathbf{x} \\
\Delta \lambda
\end{array}\right]=-\mathbf{J}_{\mathbf{m}}\left[\begin{array}{c}
\Delta \mathbf{x} \\
\Delta \lambda
\end{array}\right]=\left[\begin{array}{c}
\Delta \mathbf{G} \\
\Delta R
\end{array}\right]
$$

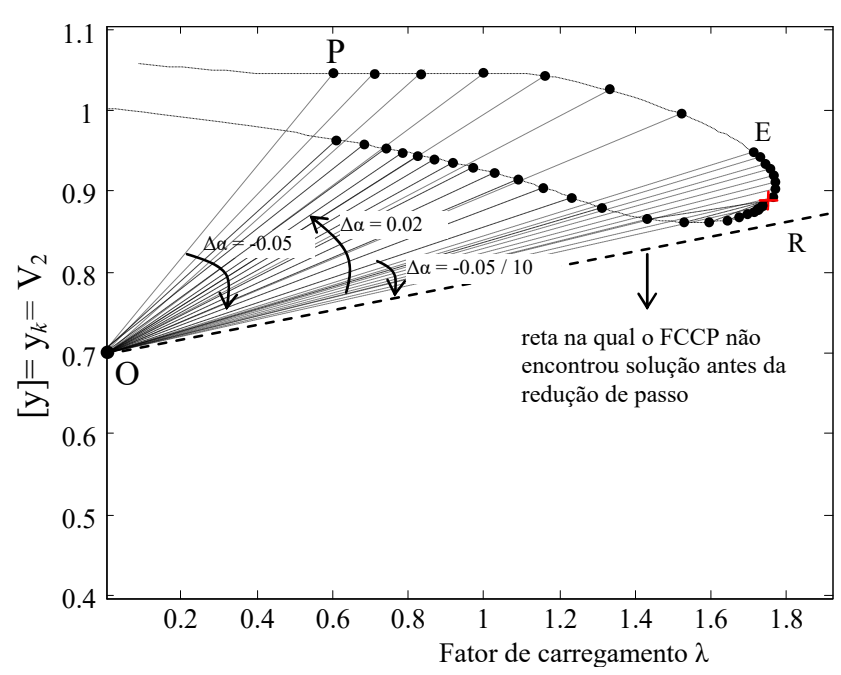

Figura 1. Curva $\lambda-y_{k}$ da barra 2 do sistema IEEE 14

sendo que $\mathbf{x}=\left[\boldsymbol{\theta}^{\mathrm{T}} \mathbf{V}^{\mathrm{T}}\right]^{\mathrm{T}}, \mathbf{J}$ e $\mathbf{J}_{\mathbf{m}}$ são as matrizes Jacobianas do FC convencional e do FCC, e $\mathbf{G}_{\lambda}$ corresponde à derivada de $\mathbf{G}$ em relação a $\lambda$.

Diminuindo-se gradualmente o valor de $\alpha, \alpha^{i+1}=\alpha^{i}-\Delta \alpha$ obtém os próximos pontos da curva $\lambda$-[y], até que o FCCP não apresenta mais solução, ou seja, quando a reta $R$ não mais intercepta a trajetória de soluções. Nesse ponto, mudam-se as coordenadas do centro do feixe de retas do ponto "O" para o ponto médio (PM) situado entre os dois últimos pontos obtidos, ou seja, as coordenadas do novo centro de feixe de retas serão PM $\left(\left(\|\mathbf{y}\|_{3}{ }^{a}+\|\mathbf{y}\|_{3}{ }^{b}\right) / 2,\left(\lambda^{a}+\lambda^{b}\right) / 2\right)$. A seguir, considere a equação da reta que passa por PM e pelo último ponto obtido. Uma vez passado o PMC, o restante da curva é obtida considerando-se novamente as equações das retas que passam pelas coordenadas do ponto "O", com $\Delta \alpha=-\Delta \alpha$. Em alguns sistemas é possível obter o PMC usando-se apenas as equações das retas pertencentes ao feixe que passa pelo ponto "O", como apresentado na Fig. 1. Resultados similares podem ser observados na Fig. 2, neste caso, considera-se o vetor $[\mathbf{y}]=\theta_{k}$, onde $k$ representa a barra crítica 14 e o ponto escolhido "O" como falado anteriormente, com coordenadas iguais a do caso base para $\theta_{k}$ e fator de carregamento nulo. $\mathrm{Na}$ Fig. 2(a), a curva P-V completa pode ser obtida pelo FCCP sem problemas de singularidade da matriz $\mathbf{J}_{\mathbf{m}}$, o mesmo não ocorre com o FC que é singular próximo ao PMC. O determinante da matriz $\mathbf{J}$ e $\mathbf{J}_{\mathbf{m}}$ podem ser vistos na Fig. 2(c) em que comprovam os resultados da singularidade. O número de iterações para ambos os métodos são mostrados na Fig. 2(b). 

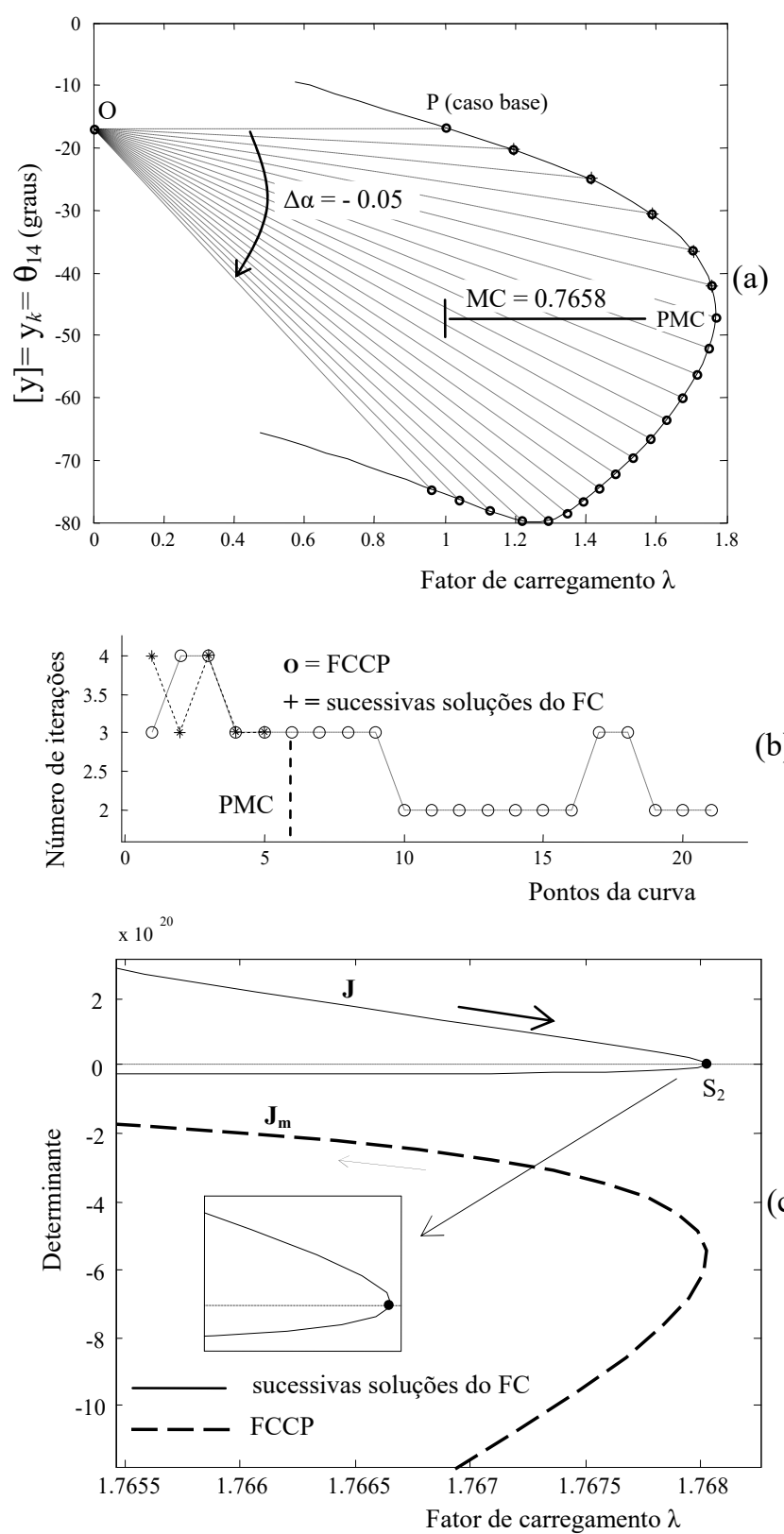

Figura 2. Desempenho do FCCP para o sistema IEEE-14 barras: (a) ângulo da tensão da barra crítica $\left(\theta_{14}\right)$ em função de $\lambda$, (b) número de iterações para 0 FCCP e Newton convencional, (c) determinante das matrizes $\mathbf{J}$ e $\mathbf{J}_{\mathbf{m}}$.

Para facilitar a compreensão do FCCP, as Fig. 3 e 4 apresentam os fluxogramas correspondentes e a Fig. 5 está representando o fluxograma dos procedimentos P1 e P2 respectivamente. O fluxograma apresentado na Fig. 5 correspondente ao plano $\lambda-\theta$ e ficou restrito apenas no primeiro feixe de retas. Nota-se na Fig. 5, que se não houver mudança significativa no sistema (tipo da barra PV para PQ em virtude da violação de seus limites) não se atualiza a matriz $\mathbf{J}_{\mathbf{m}}$ que corresponde ao sistema matricial de equações (6).

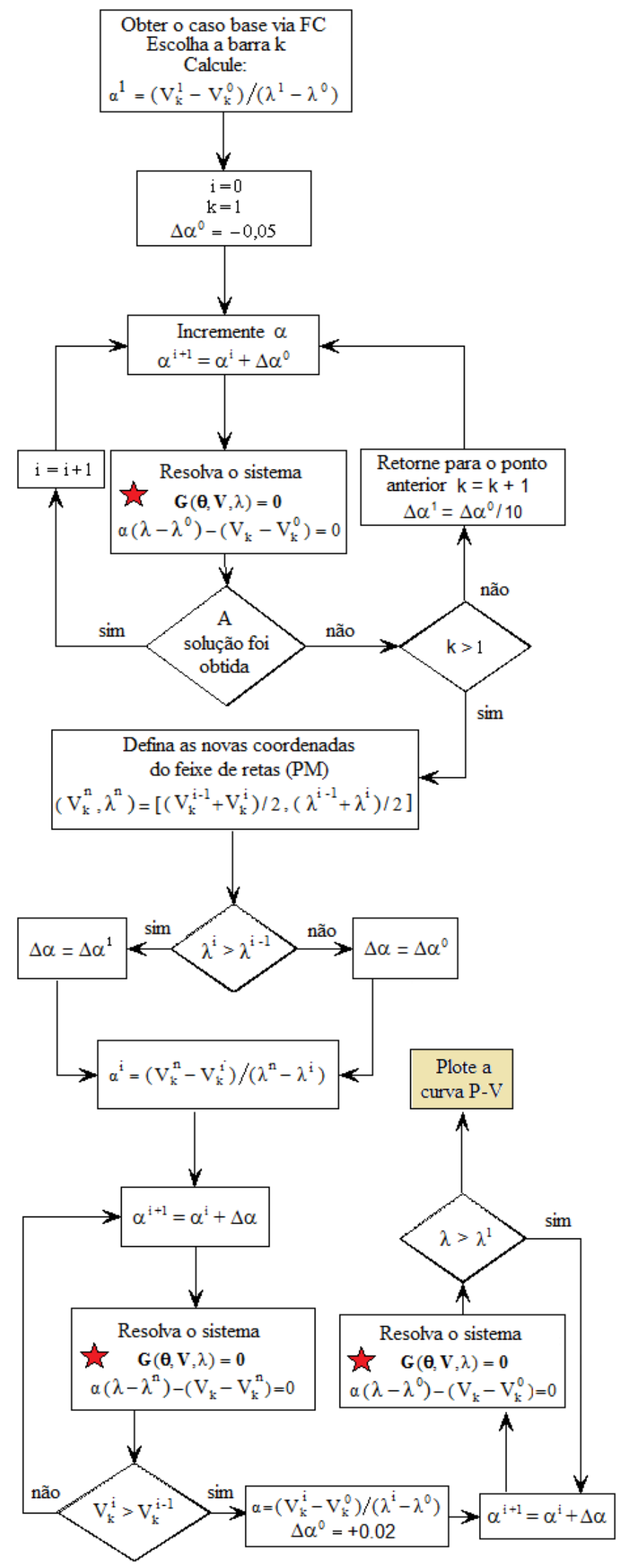

Figura 3. Fluxograma do FCCP utilizando o plano $\lambda-\mathrm{V}$. 


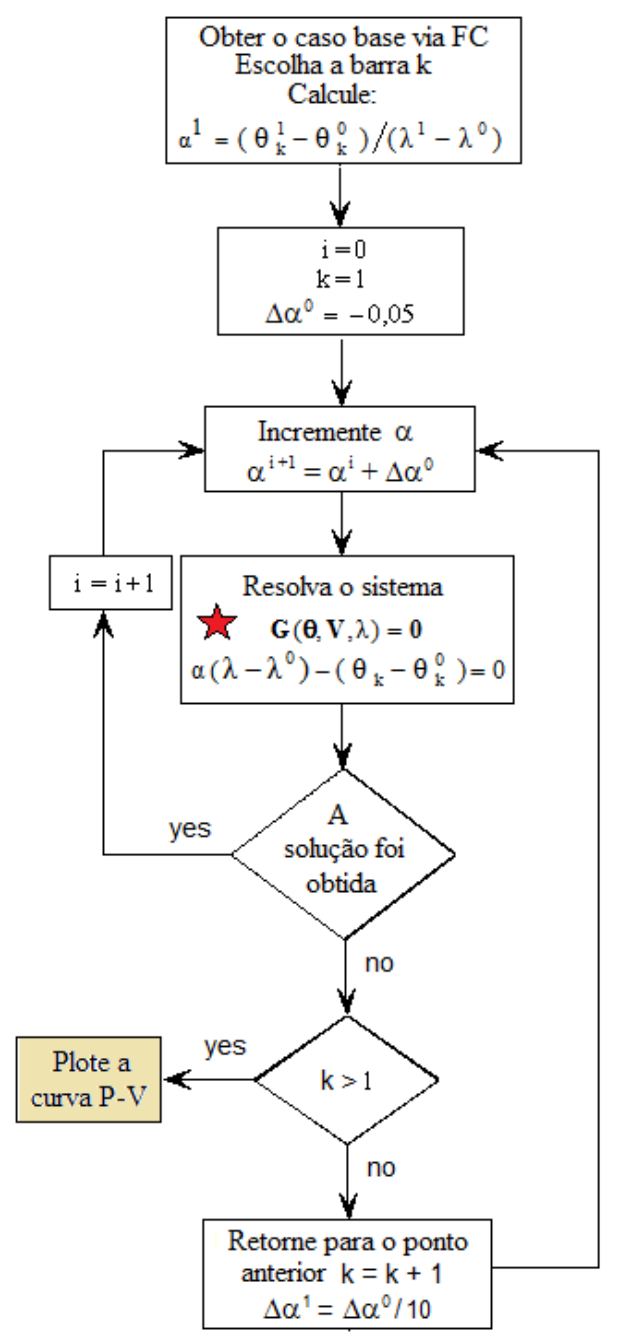

Figura 4. Fluxograma do FCCP utilizando o plano $\lambda-\theta$.

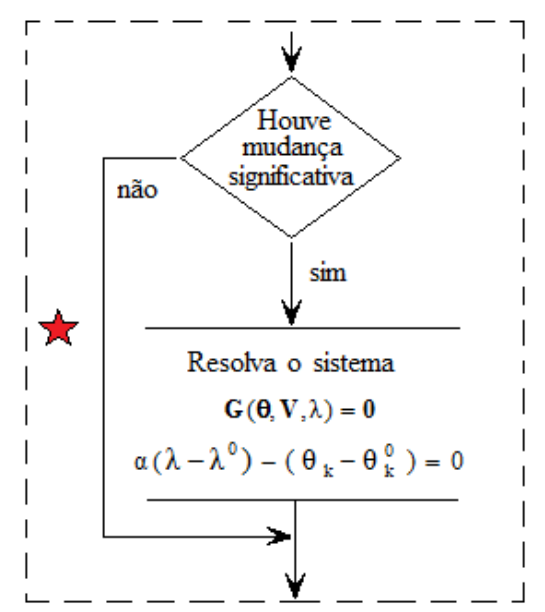

Figura 5. Fluxograma para atuação dos limites para especificar P1 e P2.

\section{RESULTADOS}

A tolerância adotada para o mismatch total de potência em todos os testes realizados foi de $10^{-4}$ p.u. Da mesma forma que no método convencional de $\mathrm{FC}$, foi feito a consideração dos limites de potência reativa $(Q)$ nas barras $P V$ s. O valor adotado para o passo inicial $(\Delta \alpha)$ para ambos os planos utilizados foi de 0.05 respectivamente.

A tabela 1 apresenta, para os cinco sistemas analisados, os valores de $\lambda$ no PMC $\left(\lambda^{*}\right)$ e da tensão crítica computada pelas técnicas de parametrizações apresentadas $\left(\lambda-V_{k}\right)$ e $\left(\lambda-\theta_{k}\right)$. Todos os valores do PMC descritos na tabela foram obtidos considerando o passo de 0.05 .

A Fig. 6 mostra o desempenho FCCP durante a obtenção da curva P-V do sistema IEEE-300, considerando como parâmetro a magnitude da tensão da barra crítica 526. Da Fig. 6(a) se pode notar que no caso da barra crítica (barra 526), o algoritmo não necessita efetuar a mudança do centro do feixe de retas para o ponto médio (PM). A Fig. 6(b) mostra o número de iterações gastas pelo FCCP e pelo método do FCC parametrizado por tensão, de onde se pode notar que os desempenhos (número de iterações e tempo de CPU) para ambos os métodos foram praticamente os mesmos.

A Fig. 6(c) apresenta o desempenho entre os tempos de CPU utilizados pelo FCC parametrizado pela magnitude de tensão da barra 526, $\left(V_{526}\right)$ e pelo FCCP. Novamente aqui,
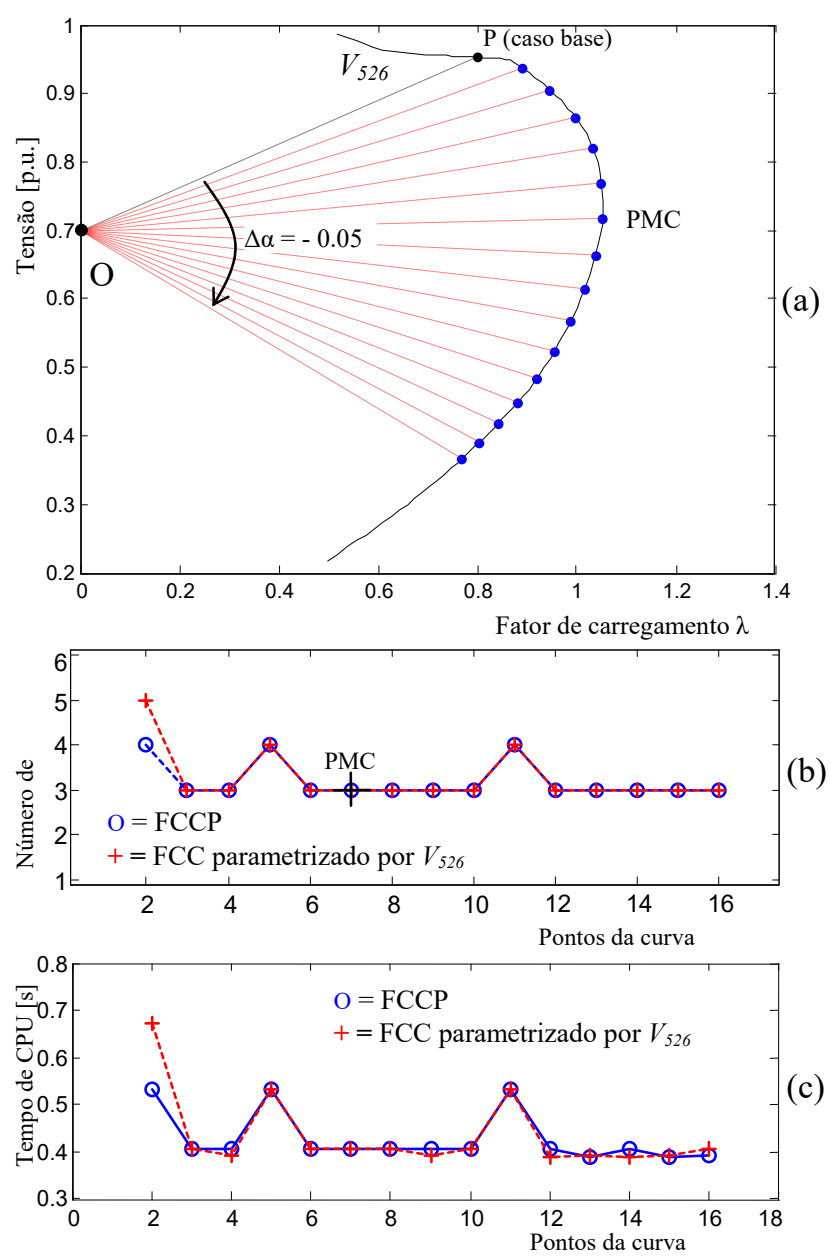

(c)

Figura 6. Desempenho do FCCP para o IEEE-300: (a) magnitude da tensão da barra de carga crítica $526\left(V_{526}\right)$ em função de $\lambda$, (b) número de iterações, (c) tempos de CPU para o FCCP e para o FCC parametrizado pela magnitude de tensão da barra 526 . 
TABELA I. PONTO DE MÁXIMO CARREGAMENTO E TENSÃO CRÍTICA DOS SISTEMAS ANALISADOS.

\begin{tabular}{|c|c|c|}
\hline Sistemas & $\lambda^{*}$ & Tensão Crítica (p.u.) \\
\hline $14^{(1)}$ & 1.7659 & 0.63 \\
\hline $118^{(1)}$ & 2.0034 & 0.86 \\
\hline $300^{(1)}$ & 1.0530 & 0.71 \\
\hline $638^{(1)}$ & 1.0087 & 0.77 \\
\hline $904^{(1)}$ & 1.1976 & 0.68 \\
\hline $14^{(2)}$ & 1.7674 & 0.59 \\
\hline$* 118^{(2)}$ & 1.8638 & 0.77 \\
\hline $300^{(2)}$ & 1.0540 & 0.71 \\
\hline $638^{(2)}$ & 1.0087 & 0.78 \\
\hline $904^{(2)}$ & 1.1988 & 0.66 \\
\hline
\end{tabular}

pode-se considerar que a diferença de tempo entre ambos os métodos é praticamente desprezível, isto é, que os desempenhos dos métodos são praticamente iguais quando se trata da atualização da matriz $\mathbf{J}$ a cada iteração.

\subsection{Influência da atualização da Matriz Jacobiana}

O objetivo aqui é o de avaliar o FCCP, comparando seus desempenhos considerando dois procedimentos utilizados. No primeiro procedimento (P1) a matriz Jacobiana é atualizada a cada iteração e no segundo procedimento (P2), J é atualizada somente quando o sistema sofrer mudanças significativas.

Para todas as tabelas a seguir, para ambos os procedimentos, é apresentado o número total de iterações (IC) necessários para o traçado completo da curva $\mathrm{P}-\mathrm{V}$, e no caso do procedimento $\mathrm{P} 2$, também é mostrado o número total de iterações (ACo) para o qual há a atualização da matriz. São apresentados na sétima coluna de suas respectivas tabelas os tempos computacionais requeridos por cada uma das técnicas de parametrização analisadas, considerando o procedimento P2. Os valores foram normalizados pelos respectivos tempos requeridos considerando o procedimento $\mathrm{P} 1$, o qual se encontra na quarta coluna de cada uma das tabelas.

Resultados do desempenho do sistema IEEE-118 barras são apresentados na Fig. 7. A Fig. 7(a) apresenta a curva P-V (curva da barra crítica $V_{13}$ em função do carregamento $\lambda$ ). É apresentado o número de iterações, considerando os procedimentos P1 e P2 na Fig. 7(b). Em termos de tempo de CPU, embora o número de iterações pareça maior para o procedimento $\mathrm{P} 2$, apresenta-se o contrário, isto se deve ao fato que pelo procedimento P2 a matriz é atualizada somente quando necessário. Estes resultados são comprovados na Fig. 7(c) e na tabela 2, como pode ser visto, o procedimento P1 exige 94 iterações (com atualizações em todas elas), enquanto que o P2 exige 108 iterações com apenas 59 atualizações, acarretando com isso, uma redução em média de $29.4 \%$ no tempo de CPU.

$\mathrm{Na}$ Fig. 8 e na tabela 2 apresentam-se o desempenho do FCCP para o sistema localizado no sudoeste americano de 904 barras. Na Fig. 8(a) encontra-se a curva P-V da barra crítica (138) em função de $\lambda$. Na Fig. 8(b) são apresentados os números de iterações (IC) para cada um dos procedimentos,
P1 e P2. São apresentados na Fig. 8(c) os tempos de CPU necessários para obtenção de cada ponto da curva, utilizando ambos os procedimentos.

Ao observar a tabela 2, novamente nota-se uma redução do tempo computacional utilizando o procedimento $\mathrm{P} 2$, embora o número de iterações seja maior (79), a matriz Jacobiana foi atualizada somente 32 vezes (ACo), enquanto que com P1, foram feitas ao todo 75 atualizações. Assim, há uma redução de $29.2 \%$ no tempo de CPU. Já para os sistemas IEEE 300, o sistema Sul-Sudeste Brasileiro de 638 barras e o de 904 barras, a redução do tempo computacional para o traçado completo da curva P-V das barras críticas foram de $39.3 \%$, $41.1 \%$ e $29.2 \%$, respectivamente.

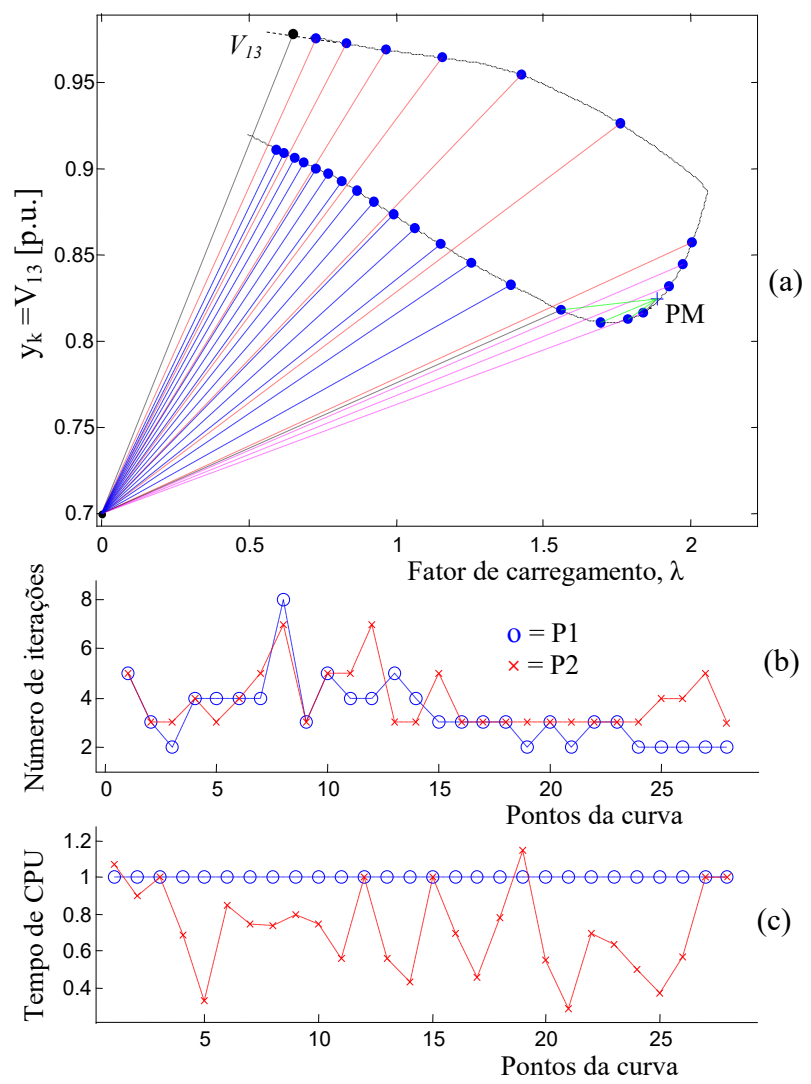

Figura 7. Sistema 118 barras: (a) curva P-V da barra crítica 13, (b) número de iterações, (c) tempo de CPU.

TABELA II. DESEMPENHO DO FCCP CONSIDERANDO A BARRA CRÍTICA E OS PROCEDIMENTOS P1 E P2 COM PLANO $\lambda-V_{k}$

\begin{tabular}{cccccccc}
\hline & & \multicolumn{3}{c}{ P1 } & \multicolumn{4}{c}{ P2 } \\
Sistemas & Barra & IC & $\begin{array}{c}\text { Tempo } \\
\text { CPU (p.u.) }\end{array}$ & IC & ACo & $\begin{array}{c}\text { Tempo } \\
\text { CPU (p.u.) }\end{array}$ & $\begin{array}{c}\text { Redução } \\
\text { CPU (\%) }\end{array}$ \\
\hline 14 & 14 & 86 & 1.000 & 166 & 15 & 0.789 & 21.1 \\
118 & 13 & 94 & 1.000 & 108 & 59 & 0.706 & 29.4 \\
300 & 526 & 66 & 1.000 & 69 & 27 & 0.607 & 39.3 \\
638 & 150 & 92 & 1.000 & 135 & 26 & 0.589 & 41.1 \\
904 & 138 & 75 & 1.000 & 79 & 32 & 0.708 & 29.2 \\
\hline
\end{tabular}

Os resultados considerando uma barra $k$ qualquer no plano $\lambda-V_{k}$ são apresentados na tabela 3. Considerando como exemplo a barra 44 do sistema teste do IEEE-118, pode se notar uma redução do tempo computacional utilizando o procedimento $\mathrm{P} 2$. O número de iterações utilizando o 
procedimento $\mathrm{P} 1$ foi igual a 110, enquanto que para o procedimento P2 foram necessárias 130 iterações. Novamente, conforme foi falado anteriormente considerando a barra crítica para o traçado das curvas $\mathrm{P}-\mathrm{V}$ e aqui considerando uma barra $k$ qualquer, embora o número de iterações seja maior (130), a matriz Jacobiana foi atualizada somente 54 vezes (ACo). Assim, há uma redução de $24,4 \%$ no tempo de CPU. Já para os demais sistemas 300, 638 e 904 barras, as reduções de tempo computacional foram de $21,1 \%, 39,2 \%$ e $23.7 \%$, respectivamente.

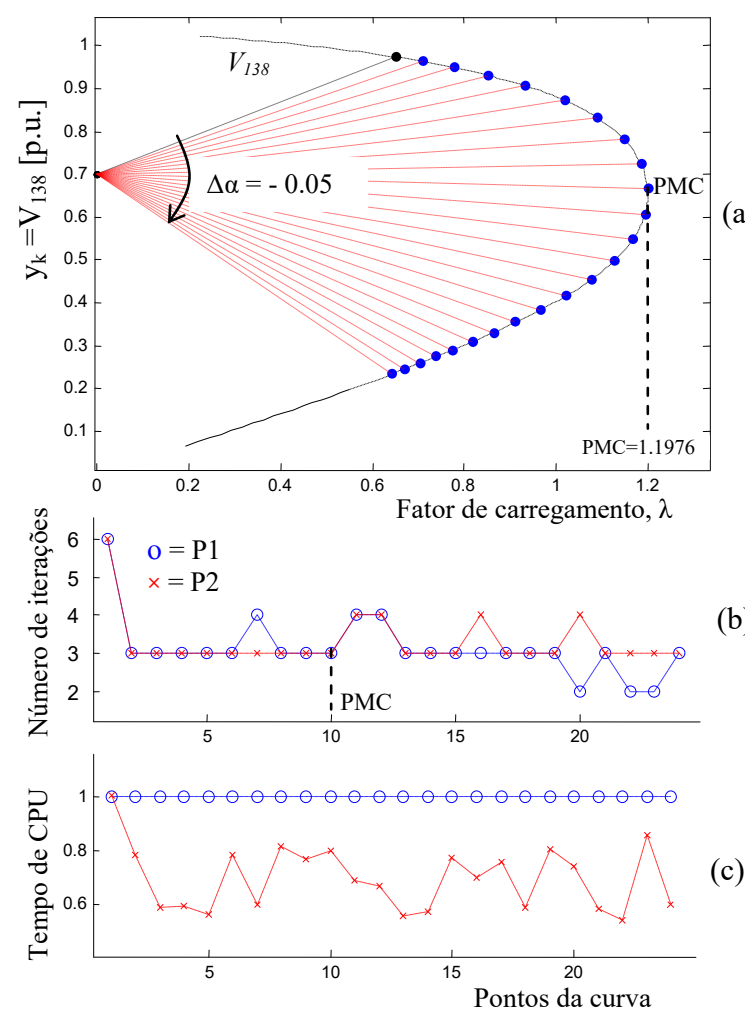

Figura 8. Sistema 904 barras: (a) curva P-V da barra crítica 138, (b) número de iterações, (c) tempo de CPU.

TABELA III. DESEMPENHO DO FCCP CONSIDERANDO UMA BARRA $K$ QUALQUER E OS PROCEDIMENTOS P1 E P2 COM PLANO $\lambda-V_{k}$

\begin{tabular}{|c|c|c|c|c|c|c|c|}
\hline \multirow[b]{2}{*}{ Sistemas } & \multirow[b]{2}{*}{ Barra } & \multicolumn{2}{|r|}{ P1 } & \multicolumn{3}{|c|}{ P2 } & \multirow[b]{2}{*}{$\begin{array}{l}\text { Redução } \\
\text { CPU (\%) }\end{array}$} \\
\hline & & IC & $\begin{array}{c}\text { Tempo } \\
\text { CPU (p.u.) }\end{array}$ & IC & $\mathrm{ACo}$ & $\begin{array}{c}\text { Tempo } \\
\text { CPU (p.u.) }\end{array}$ & \\
\hline 14 & 2 & 99 & 1.000 & 200 & 18 & 0.790 & 21.0 \\
\hline 118 & 44 & 110 & 1.000 & 130 & 54 & 0.756 & 24.4 \\
\hline 300 & 63 & 82 & 1.000 & 115 & 55 & 0.789 & 21.1 \\
\hline 638 & 199 & 90 & 1.000 & 137 & 25 & 0.608 & 39.2 \\
\hline 904 & 131 & 99 & 1.000 & 118 & 43 & 0.763 & 23.7 \\
\hline
\end{tabular}

A Fig. 9 apresenta o desempenho do FCCP utilizando o plano $\lambda-\theta_{14}$ para o sistema IEEE 14 barras. Observa-se na Fig. 9(a) o número de iterações e o tempo de CPU necessários para obter cada ponto da curva utilizando os procedimentos $\mathrm{P} 1 \mathrm{e}$ P2, também é apresentado o número de atualizações ACo. Na Fig. 9(b) é apresentado resultado similar ao da Fig. 9(a), porém utilizando a barra de carga 13 e nas Fig. 10(a) e (b) são apresentados resultados do sistema IEEE 118 para a barra crítica 52 e para a barra de carga 53 .
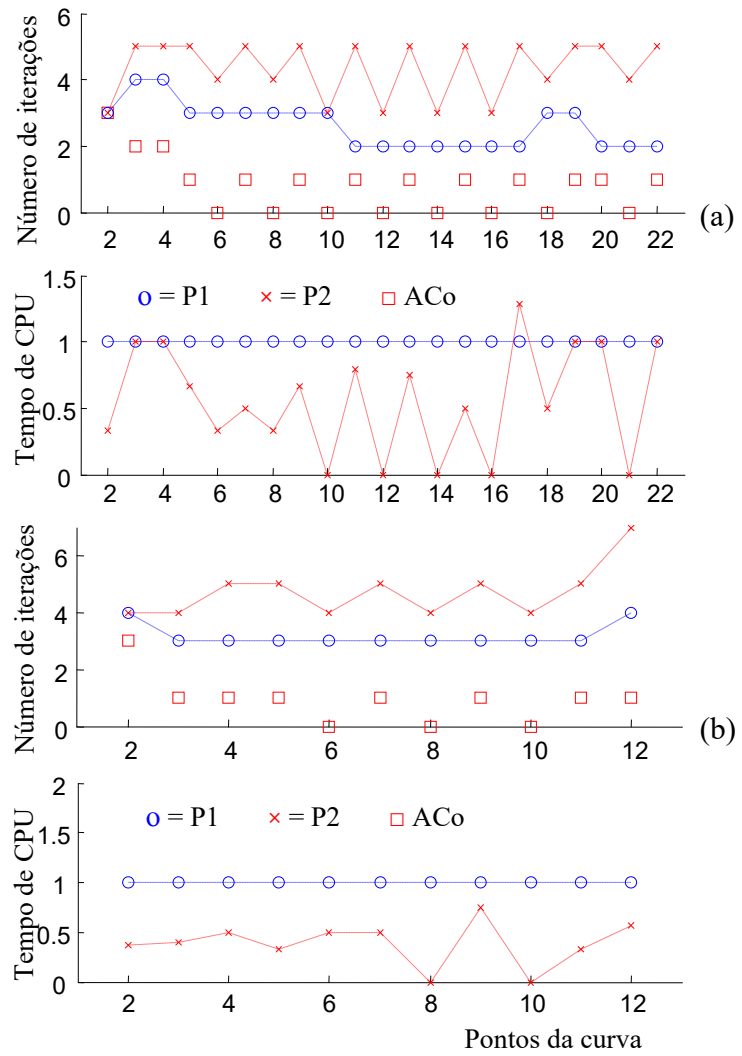

Figura 9. Desempenho do FCCP sistema IEEE 14 barras: (a) número de iterações e tempo de CPU para barra crítica 14, (b) número de iterações e tempo de CPU para barra 13.
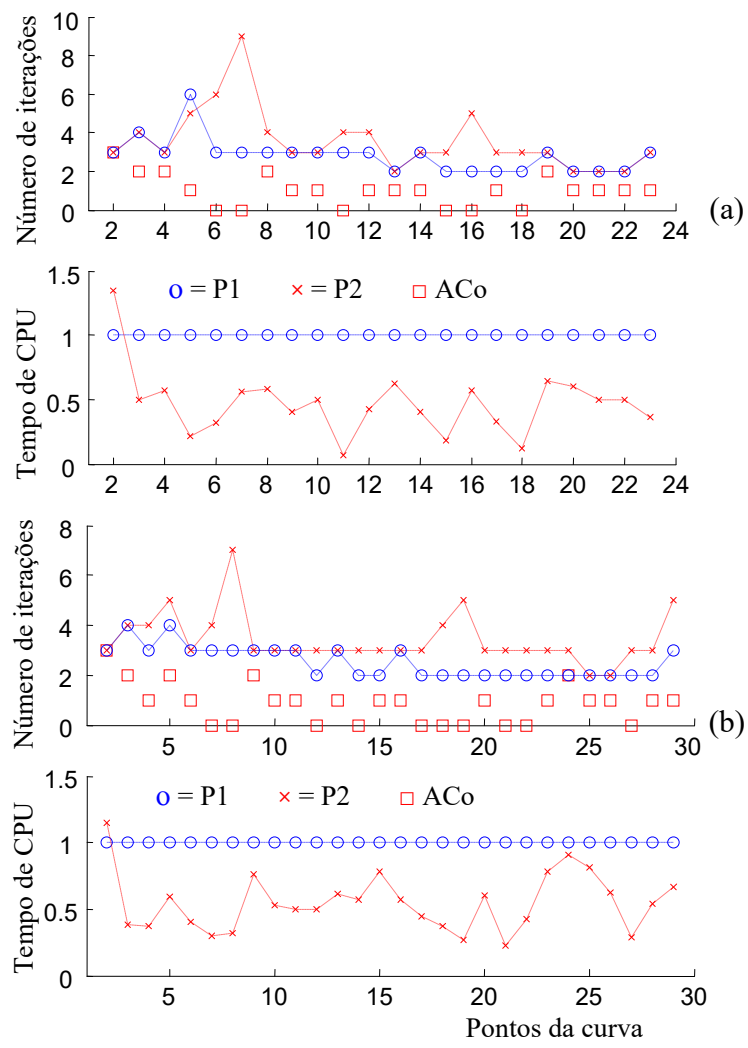

Figura 10. Desempenho do FCCP sistema IEEE 118 barras: (a) número de iterações e tempo de CPU para barra crítica 52, (b) número de iterações e tempo de CPU para barra 53. 
A Fig. 11 apresenta resultados para o sistema IEEE 300 barras. A curva trajetória de soluções bem como os pontos obtidos para o traçado da curva P-V utilizando o plano $\lambda$ - $\theta_{526}$ são mostrados na Fig. 11(a). O número de iterações utilizando os procedimentos P1 e P2, também o número de atualizações ACo e o tempo de CPU para obtenção de cada ponto da curva são mostrados na Fig. 11(b).

Resultados similares para o sistema de grande porte SulSudeste Brasileiro de 638 barras e o sistema localizado no Sudoeste Americano de 904 barras são apresentados na Fig. 12. O número de iterações utilizando os procedimentos P1 e P2, também o número de atualizações ACo e o tempo de CPU para obtenção de cada ponto da curva da barra crítica 150 e da barra 149 do sistema 638 barras são mostrados na Fig. 12(a) e (b) e para a curva da barra crítica 138 do sistema 904 barras é mostrado na Fig. 12(c).

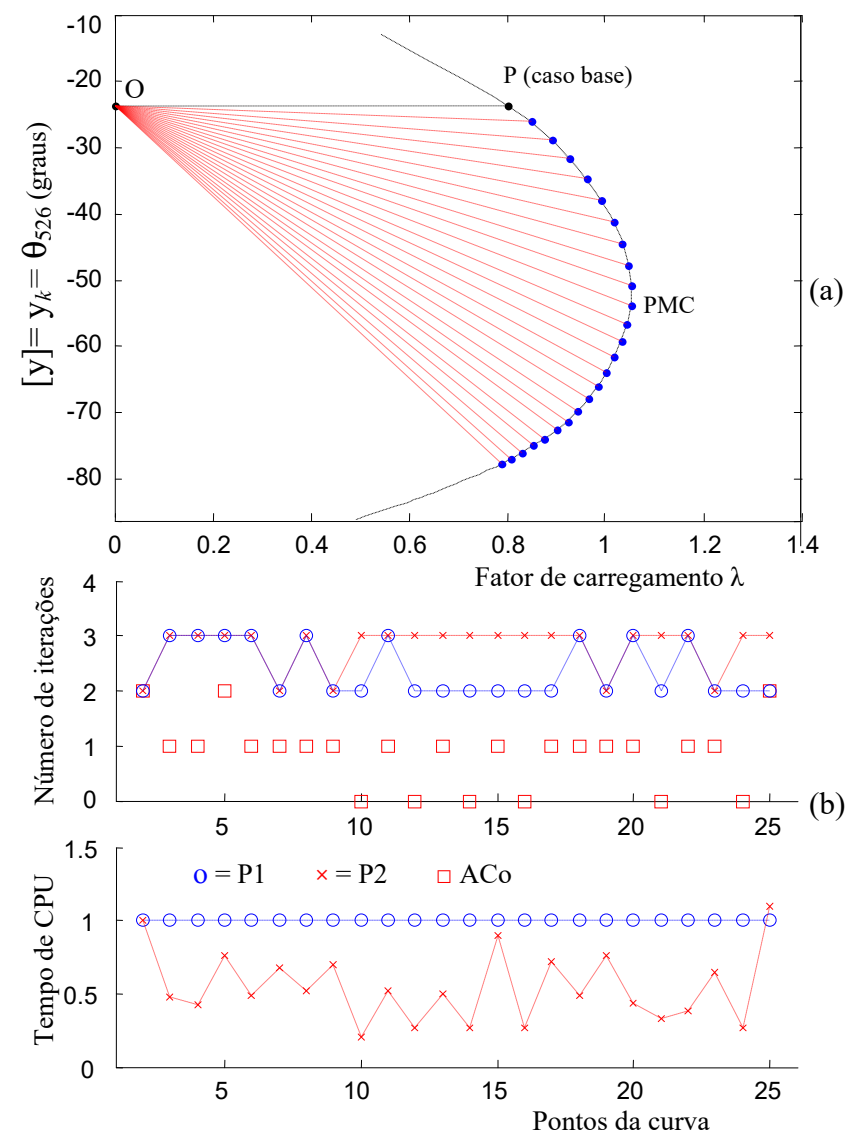

Figura 11. Desempenho do FCCP para o IEEE-300: (a) ângulo da tensão da barra de carga crítica $526\left(V_{526}\right)$ em função de $\lambda$, (b) número de iterações e tempo de CPU.

As tabelas 4 e 5 apresentam os números de iterações e o tempo de CPU para ambos os procedimentos $\mathrm{P} 1$ e $\mathrm{P} 2$ e o número de atualizações da matriz $\mathbf{J}_{\mathbf{m}}(\mathrm{ACo})$ no plano $\lambda$ - $\theta_{k}$. Observando a tabela 4 , tomando o sistema 638 barras como exemplo, pode se notar uma redução do tempo de CPU utilizando o procedimento $\mathrm{P} 2$ de $54.8 \%$, embora o número de iterações seja maior (165), a matriz $\mathbf{J}_{\mathbf{m}}$ foi atualizada somente 23 vezes (ACo), bem menor se comparado com as 82 atualizações quando se utiliza o procedimento P1. Tomando uma barra $k$ qualquer, como pode ser visto na tabela 5 . Os sistemas IEEE 14 e 118 e o sistema Sul-Sudeste Brasileiro de
638 barras apresentaram uma redução do tempo computacional para o traçado completo da curva $\mathrm{P}-\mathrm{V}$ de $61.2 \%, 46 \%$ e $51.9 \%$, respectivamente.
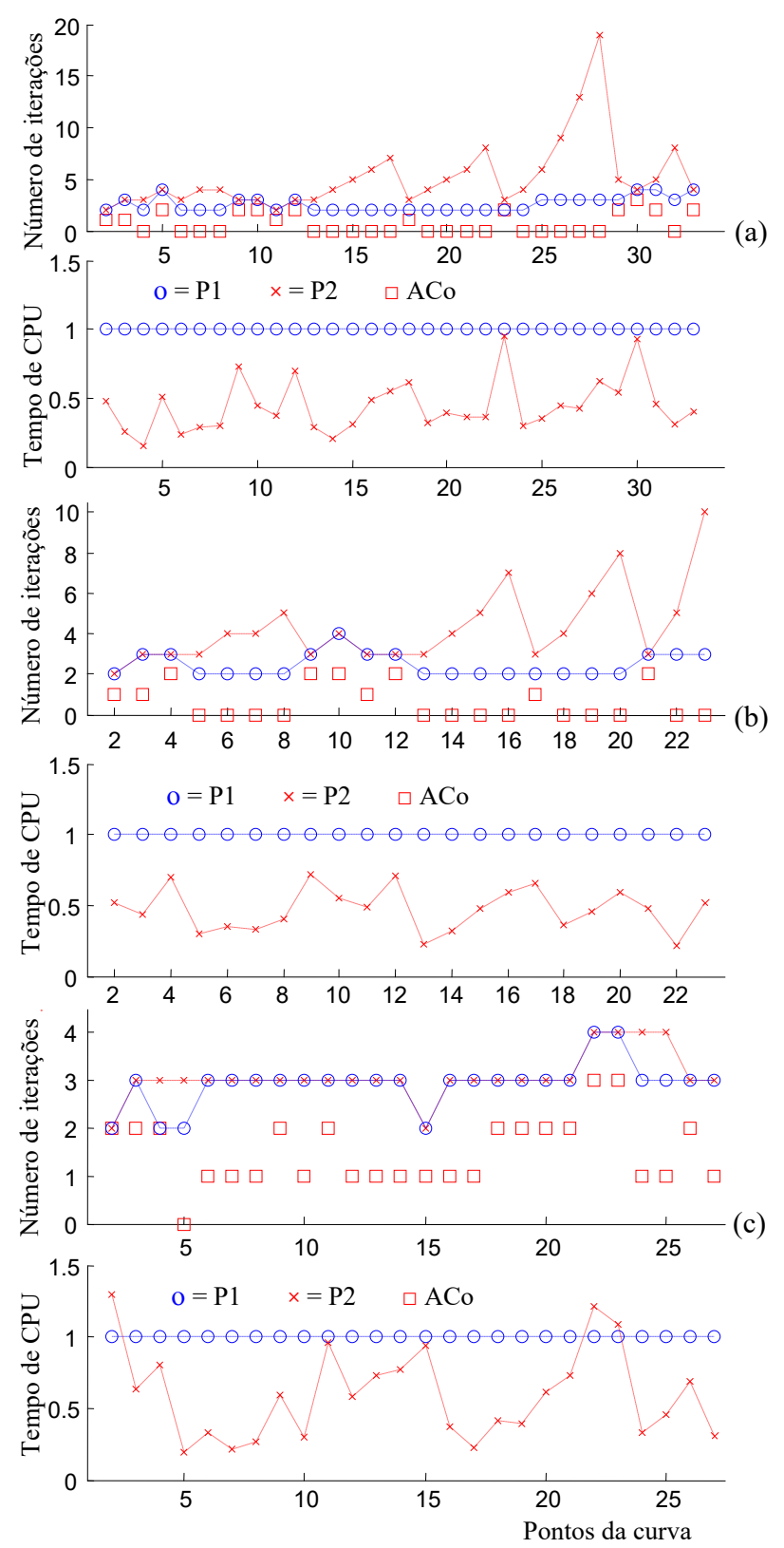

Figura 12. Desempenho do FCCP para os sistemas 638 e 904 barras: (a) número de iterações e tempo de CPU para barra crítica 150 do sistema 638 barras, (b) número de iterações e tempo de CPU para barra 149 do sistema 638 barras, (c) número de iterações e tempo de CPU para barra crítica 138 do sistema 904 barras.

TABELA IV. DESEMPENHO DO FCCP CONSIDERANDO A BARRA CRÍTICA E OS PROCEDIMENTOS P1 E P2 COM PLANO $\lambda-\theta_{k}$

\begin{tabular}{|c|c|c|c|c|c|c|c|}
\hline \multirow[b]{2}{*}{ Sistemas } & \multirow[b]{2}{*}{ Barra } & \multicolumn{3}{|c|}{$\mathrm{P} 1$} & \multicolumn{2}{|c|}{$\mathrm{P} 2$} & \multirow[b]{2}{*}{$\begin{array}{l}\text { Redução } \\
\text { CPU (\%) }\end{array}$} \\
\hline & & IC & $\begin{array}{c}\text { Tempo } \\
\text { CPU (p.u.) }\end{array}$ & IC & $\mathrm{ACo}$ & $\begin{array}{c}\text { Tempo } \\
\text { CPU (p.u.) }\end{array}$ & \\
\hline 14 & 14 & 55 & 1.000 & 91 & 17 & 0.494 & 50.6 \\
\hline *118 & 52 & 62 & 1.000 & 79 & 22 & 0.462 & 53.8 \\
\hline 300 & 526 & 57 & 1.000 & 67 & 21 & 0.542 & 45.8 \\
\hline 638 & 150 & 82 & 1.000 & 165 & 23 & 0.452 & 54.8 \\
\hline 904 & 138 & 76 & 1.000 & 80 & 39 & 0.539 & 46.1 \\
\hline
\end{tabular}

*outra configuração do sistema IEEE 118 barras 
TABELA V. DESEMPENHO DO FCCP CONSIDERANDO UMA BARRA $K$ QUALQUER E OS PROCEDIMENTOS P1 E P2 COM PLANO $\lambda-\theta_{k}$

\begin{tabular}{cccccccc}
\hline & \multicolumn{3}{c}{ P1 } & \multicolumn{4}{c}{ P2 } \\
Sistemas & Barra & IC & $\begin{array}{c}\text { Tempo } \\
\text { CPU (p.u.) }\end{array}$ & IC & ACo & $\begin{array}{c}\text { Tempo } \\
\text { CPU (p.u.) }\end{array}$ & $\begin{array}{c}\text { Redução } \\
\text { CPU (\%) }\end{array}$ \\
\hline 14 & 13 & 35 & 1.000 & 52 & 10 & 0.388 & 61.2 \\
$* 118$ & 53 & 71 & 1.000 & 96 & 24 & 0.540 & 46.0 \\
638 & 149 & 54 & 1.000 & 95 & 14 & 0.481 & 51.9 \\
\hline
\end{tabular}

*outra configuração do sistema IEEE 118 barras

\section{CONCLUSÃO}

Neste trabalho foi apresentado uma técnica de parametrização proposta (FCCP) considerando o método de Newton Desonesto e também considerando o plano $\lambda-\theta$ para o traçado completo das curvas P-V. Uma técnica conhecida como Dishonest Newton Method foi apresentada e consiste em atualizar a matriz Jacobiana somente quando o sistema sofrer alguma mudança significativa.

Dos resultados apresentados, pode-se concluir que foi possível conseguir uma redução do tempo computacional necessários para obter todo o traçado da curva $\mathrm{P}-\mathrm{V}$, isto é, uma melhora na eficiência da técnica proposta, sem a perda de robustez. Para todos os sistemas estudados, houve uma redução em média de $29 \%$ utilizando o plano $\lambda-\mathrm{V}$ e $51.3 \%$ considerando o plano $\lambda-\theta$ em termos de tempo computacional necessário para a obtenção da curva $\mathrm{P}-\mathrm{V}$. Com isso, os resultados obtidos confirmam uma melhora na eficiência do método proposto e também mostram sua viabilidade para aplicações em estudos relacionados com a avaliação da estabilidade estática de tensão.

\section{AGRADECIMENTOS}

Os autores agradecem ao $\mathrm{CNPq}$ e a Capes pelo apoio financeiro.

\section{REFERÊNCIAS}

[1] M. Abbott, "Electricity reform and gains from the reallocation of resources," The Electricity Journal, Atlanta, vol. 20, pp. 72-78, 2007.

[2] V. Ajjarapu and C.Christy. The Continuation Power Flow: a Tool for Steady State Voltage Stability Analysis. IEEE Trans. on Power Systems, vol. 7, n. 1, February, pp. 416-423, 1992.

[3] A. Bonini Neto, D. A. Alves. Técnicas de Parametrização Global para o Fluxo de Carga Continuado. Controle \& Automação (Impresso), v. 21, p. 323-337, 2010.

[4] P. Xu, X. Wang, V. Ajjarapu. Continuation power flow with adaptive stepsize control via convergence monitor. IET Generation, Transmission \& Distribution, 6(7), pp. 673-679. 2012.

[5] R. Seydel, "From Equilibrium to Chaos: Pratical Bifurcation and Stability Analisys", 2th. ed., Springer-Verlag: New York, 1994.

[6] E Garbelini, D. A. Alves, A. Bonini Neto, E. Righeto, L. C. P. da Silva, C. A. Castro. An efficient geometric parameterization technique for the continuation power flow. Electric Power Systems Research, vol. 77, pp. 71-82, 2007.

[7] E. M. Magalhães, A. Bonini Neto, D. A. Alves. A parameterization technique for the continuation power flow developed from the analysis of power flow curves. Mathematical Problems in Engineering, vol. 2012, pp. 1-24, 2012.
[8] A. Bonini Neto, D. A. Alves. Improved geometric parameterization techniques for continuation power flow. IET Generation, Transmission \& Distribution; vol. 4, pp. 1349-1359. 2010.

[9] A. Bonini Neto, D. A. Alves. Técnica de Parametrização Geométrica para o Fluxo de Carga Continuado Baseado nas Variáveis Tensão Nodal e Fator de Carregamento. SBA. Sociedade Brasileira de Automática. , v.19, p.350 - 366. 2008.

[10] V. Ajjarapu. Computational Techniques for Voltage Stability Assessment and Control. Power Electronics and Power Systems Series, Springer. 2010.

[11] D. A. Alves, L. C. P. da Silva, C. A. Castro, V. F. da Costa. Study of alternative schemes for the parameterization step of the continuation power flow method based on physical parameters, part I: mathematical modeling. Electric Power Components and Systems, vol. 31, pp. 11511166. 2003.

[12] H. Sheng, H.D. Chiang. CDFLOW: Practical tool for tracing stationary behaviors of general distribution networks. IEEE Trans. on Power Systems, 29(3), pp.1365-1371. 2014.

[13] P. Zhu, G. Taylor, M. Irving. Performance analysis of a novel Q-limit guided continuation power flow method, IET Generation, Transmission \& Distribution, 3(12), pp. 1042-1051. 2009.

[14] M. Abdel-Akher. Voltage stability analysis of unbalanced distribution systems using backward/forward sweep load-flow analysis method with secant predictor. IET Generation, Transmission \& Distribution; 7(3), pp. 309-317. 2013.

[15] M. Abdel-Akher, M. M. Aly, Z. Ziadi, H. El-kishky, M. Abdel-Warth. Voltage stability modeling and analysis of unbalanced distribution systems with wind turbine and energy systems. In:Industrial Technology (ICIT), 2014 IEEE International Conference on. IEEE, Seoul, Korea, pp. 565-570, 2014.

[16] A. Pama, G. Radman. A new approach for estimating voltage collapse point based on quadratic approximation of PV curves. Electric Power System Research; 79, pp. 653-659. 2009.

[17] F. Karbalaei, S. Abasi. A prediction of voltage collapse in presence of voltage dependent loads by PV curve approximation. In: Power and Engineering Conference. Proceedings...Wuhan: [s.n], v. 79, p. 653-659, 2011.

[18] H. Mori, K. Seki. Continuation Newton-GMRES power flow with linear and nonlinear predictors. Power Engineering, 2007 Large Engineering Systems Conference, Proceedings...Tokio: [s.n], p. 171 - 175, 2007.

[19] S. H. Li, H. D. Chiang. Nonlinear predictors and hybrid corrector for fast continuation power flow. IET Generation, Transmission \& Distribution; 2, pp. 341-354. 2008.

[20] T. Kojima, H. Mori. Development of nonlinear predictor with a set of predicted points for continuation power flow. Electrical Engineering in Japan, 163(4), pp. 30-41. 2008.

[21] H. Mori, K. Seki. Non-linear-predictor-based continuation power flow for unbalanced distribution systems. Transmission \& Distribution Conference \& Exposition: Asia and Pacific, [s.n], p. 1 - 4, 26-30 Oct. 2009.

[22] R. R. Matarucco, A. Bonini Neto, D. A. Alves. Assessment of branch outage contingencies using the continuation method. International Journal of Electrical Power \& Energy Systems, v. 55, p. 74-81, 2014.

[23] H. D. Chiang, A. J. Flueck, K. S. Shah, and N. Balu. "CPFLOW: A practical tool for tracing power systems steady-state stationary behavior due to load and generation variations", IEEE Trans. Power Systems, vol. 10, pp. 623-634. 1995.

[24] A. Semlyen, F. Léon. Quasi-newton power flow using partial jacobian updates. IEEE Trans. Power Syst. 16, (3), pp. 332-339. 2001.

[25] Y. Mansour, "Suggested Techniques for Voltage Stability Analysis", IEEE Power Engineering Subcommittee Report 93TH0620-5- PWR, 1993.

[26] T. Van Cutsem, C. Vournas. Voltage Stability of Electric Power Systems. Power Electronics and Power Systems International Series in Engineering and Computer Science, Springer, 2007

[27] D. Khaniya. Development of Three Phase Continuation Power Flow for Voltage Stability Analysis of Distribution System. Electrical and Computer Engineering, Mississippi State University, 2008.

[28] M. Crow. Computational Methods for Electric Power Systems. CRC Press, 2009. 


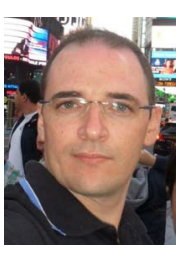

A. Bonini Neto, received his B.S. degree in Mathematics from UNIFADRA, Brazil, in 2002, his M.S. degree in Electrical Engineering from UNESP in 2006 and Ph.D. degree in Electrical Engineering from UNESP in 2011. His research interest is in the areas of mathematic and parameterization techniques applied to continuation power flow. He is with São Paulo state university - UNESP since 2014, where he is currently an assistant professor.

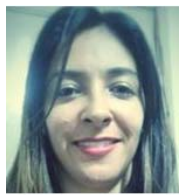

E. M. Magalhães, received his B.S. degree in Mathematics from UFMS, Brazil, her M.S. degree in Electrical Engineering from UNESP in 2011 and Ph.D. degree in Electrical Engineering from UNESP in 2015. Her research interest is in the areas of mathematic and parameterization techniques applied to continuation power flow.

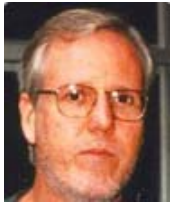

D. A. Alves, received his B.S. degree from USP, Brazil, in 1980, and his M.S. and Ph.D. degrees from UNICAMP in 1988 and 2000, respectively. His present research interest is on static voltage stability analysis. He is with UNESP since 1991 , where he is currently an assistant professor. 